\title{
NUMERICAL LOSS ANALYSIS ON SLOT-TYPE CASING TREATMENT IN A TRANSONIC COMPRESSOR STAGE
}

\author{
Ming-Min Zhu, XiaO-Qing Qiang, Jin-Fang Teng \\ School of Aeronautics and Astronautics, Shanghai Jiao Tong University, Shanghai, China \\ e-mail: jackzhushen@sjtu.edu.cn
}

To understand the loss mechanism of slot-type casing treatment, a numerical loss analysis has been carried out in a 1.5 axial transonic compressor stage with various slots. Spanwise and streamwise distribution curves of pitch-averaged entropy have been presented to survey the development of loss generation. Further, detailed entropy distributions at eight axial cuts, which have been taken through the blade row and slots, have been further analyzed to interpret the loss mechanism. The most dramatic loss growth occurred above $95 \%$ span, which directly resulted from slots injection flow upstream the leading edge. Loss generations with smooth casing have been primarily ascribed to low-momentum tip leakage flow/vortex and suction surface separation at the leading edge. CU0 slot, the arc-curved slots with $50 \%$ rotor tip exposure, has been capable of suppressing the suction surface separation loss. Meanwhile, accelerated tip leakage flow brought about additional loss near the casing and pressure surface. Upstream high entropy flow would be absorbed into the rear portion of slots repeatedly, which resulted in further loss.

Keywords: axial compressor, numerical simulation, slot-type casing treatment, loss mechanism, tip leakage flow

\section{Nomenclature}

\begin{tabular}{|c|c|c|}
\hline $\mathrm{SC} / \mathrm{CT}$ & - & smooth casing/casing treatment \\
\hline $\mathrm{AX}, \mathrm{BE}, \mathrm{CU}$ & - & axial, bend, arc-curve skewed slot, respectively \\
\hline$c_{x, t i p}$ & - & rotor tip axial chord \\
\hline $\mathrm{LE} / \mathrm{TE}$ & - & leading edge/trailing edge \\
\hline$G$ & - & mass flow, $[\mathrm{kg} / \mathrm{s}]$ \\
\hline$\pi^{*}$ & - & total pressure ratio, $[-]$ \\
\hline$\eta^{*}$ & - & isentropic efficiency, $[-]$ \\
\hline SCPE & - & peak efficiency point with smooth casing \\
\hline SCNS & - & near stall point with smooth casing \\
\hline $\mathrm{SM}$ & - & stall margin \\
\hline Eff & - & isentropic efficiency \\
\hline $\mathrm{SS} / \mathrm{PS}$ & - & suction surface/pressure surface \\
\hline$\Delta S$ & - & entropy increase relative to incoming flow, $[\mathrm{J} /(\mathrm{kg} \cdot \mathrm{K})]$ \\
\hline$\Delta S_{r e f, s c}$ & - & the reference entropy value, $[\mathrm{J} /(\mathrm{kg} \cdot \mathrm{K})]$ \\
\hline $\bar{G}_{\text {slot }}$ & - & normalized mass flow through slot opening surface, $[-$ \\
\hline
\end{tabular}

\section{Introduction}

The axial slot is a preferable configuration of casing treatment with favorable ability of enhancing the compressor operating range. Although the effect of casing treatment has a strong correlation with a specific compressor type and a flow field pattern, a rationally designed slot-type 
configuration always achieves higher stall margin improvement than a circumferential groove, ranging from 10\% to 20\% (Moore et al., 1971; Takata and Tsukuda, 1977). However, slot casing treatment also results in a much higher efficiency loss. According to the research work by Fujita and Takata (1984), a certain amount of loss in efficiency is inevitable in order to obtain desirable stall margin improvement. Particularly superior configuration does not exist.

Increasing performance requirements for aero engine demands less efficiency loss. Improving the stall margin with a serious drop in the efficiency is unacceptable. Researchers continue further investigations on configurations and design parameters of slot-type casing treatment to minimize the loss in efficiency. Arc-curve skewed slot (Yu et al., 2002) and bend skewed slot (Zhu and $\mathrm{Chu}, 2005)$ have been proved to achieve favorable stability enhancement with acceptable minor loss in certain circumstances. Some configurations even slightly increase the efficiency in specific cases (Alone et al., 2014; Li et al., 2012; Tuo et al., 2011). Researches working on design parameters presented that the efficiency loss caused by slot treatment is sensitive to slots depth, casing porosity and blade tip axial chord exposure, especially the last one. Lu et al. (2006) and Danner et al. (2009) both demonstrated that reduction in rotor exposure is conducive to minimizing the efficiency loss.

To design casing treatment with a low level loss, it is urgent to master the loss mechanism of slot treatment. Injection flow from slot casing treatment is the primary source of compressor loss (Fan et al., 2008). This paper is committed to clarifying the influence of injection flow on the compressor efficiency and elaborating the tip loss mechanism. Entropy distributions are utilized to survey the loss generation development by casing treatment along spanwise and streamwise directions. Entropy distributions at several axial cuts are examined in detail to interpret the loss development procedure.

\section{Descriptions of the investigated model and numerical method}

\subsection{Compressor stage and slot-type casing treatment}

The 1.5 stage axial transonic compressor consists of variable inlet guide vanes (IGV), rotors (R1) and stators (S1). Figure 1 shows a schematic of the compressor stage. Table 1 provides an overview of the main geometric/operational parameters of this compressor stage. The mean aspect ratio of $\mathrm{R} 1$ blades measures 1.4 with a hub-to-tip ratio of 0.7 . The solidity at the rotor tip is 1.1 and the clearance size comes to $0.3 \%$ span. The stage design total pressure ratio is 1.5 . At the design rotating speed, the rotors are characterized by the inlet relative Mach number of 1.2 corresponding to a blade tip velocity of approximately $380 \mathrm{~m} / \mathrm{s}$. While at the specified speed discussed in this paper, the maximum Mach number at the rotor tip is just less than 1.0.

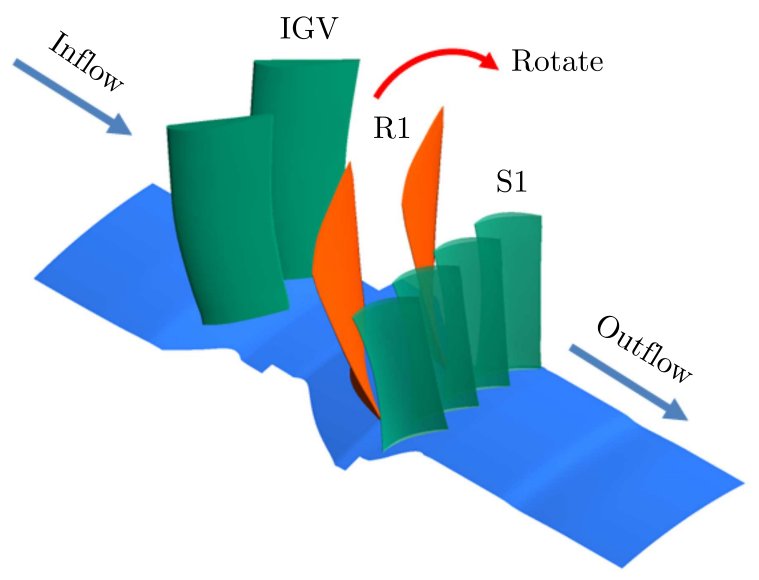

Fig. 1. Schematic of the compressor stage 
Table 1. Main geometric/operational parameters of the compressor stage

\begin{tabular}{|l|c|}
\hline \multicolumn{1}{|c|}{ Parameter [unit] } & Value \\
\hline \hline Mean aspect ratio [-] & 1.4 \\
\hline Hub-to-tip ratio [-] & 0.7 \\
\hline Rotor solidity at tip [-] & 1.1 \\
\hline Relative tip gap [\% of blade height] & 0.3 \\
\hline Stage design total pressure ratio [-] & 1.5 \\
\hline Rotor tip velocity at the design speed [m/s] & 380 \\
\hline
\end{tabular}

Three slot-type casing treatments (Notated as CT) have been designed for this compressor stage. They are an axial skewed slot, bend skewed slot and arc-curve skewed slot (notated as $\mathrm{AX}, \mathrm{BE}$ and $\mathrm{CU}) . \mathrm{BE}$ and $\mathrm{CU}$ slot can be treated as variants of $\mathrm{AX}$ slot. Three baseline slots with default design parameters are denoted as AX0, BE0 and CU0, respectively, as shown in Fig. 2.

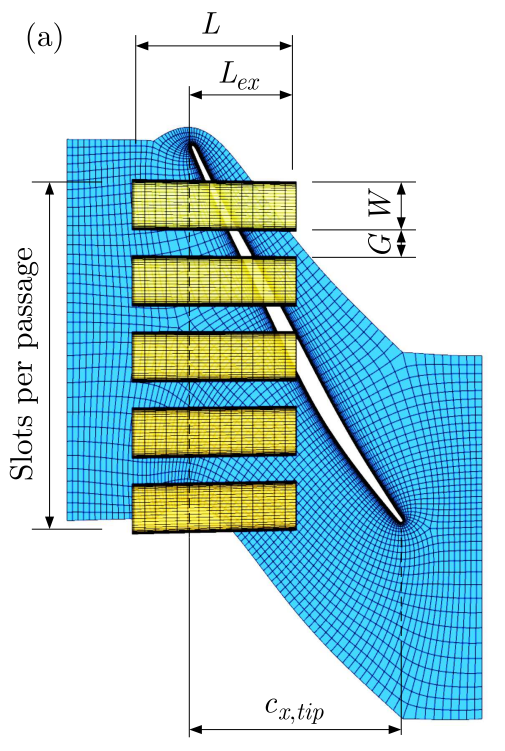

(d)

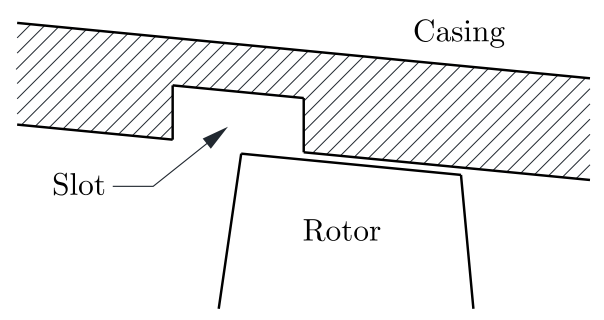

(b)

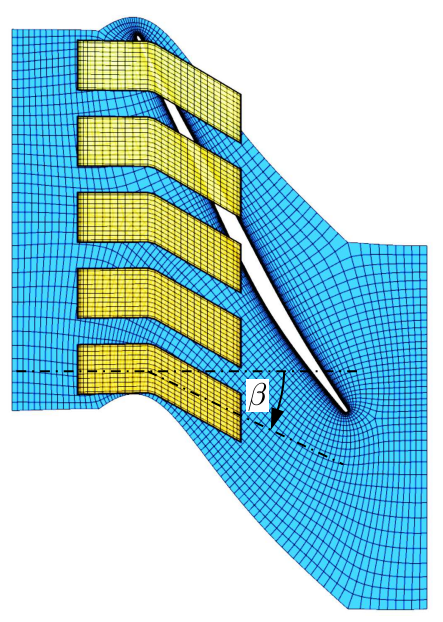

(e)

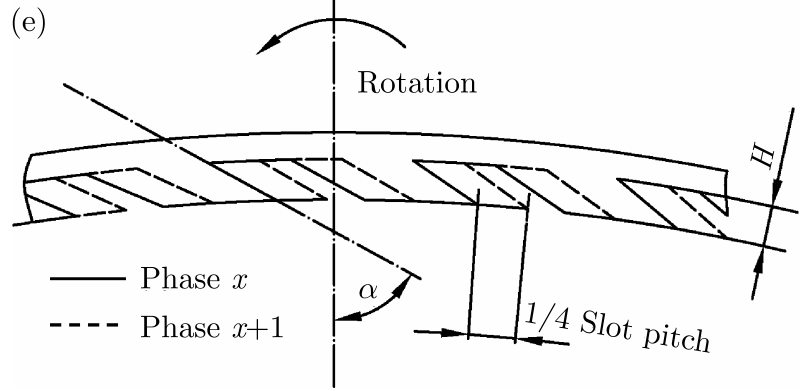

Fig. 2. Schematics of three baseline slots

All slots start from $26 \%$ of the tip axial chord $c_{x, t i p}$ upstream the leading edge (LE) in the axial direction, and its radial depth $H$ is restricted to $21.5 \% c_{x, t i p}$. The slots are parallel to the rotation axis of the rotor and are inclined by $60^{\circ}$ against a meridional plane in the direction of blade rotation, as shown in Fig. 2d. Baseline slots extend to $50 \% c_{x, t i p}$ downstream LE, and five slots are evenly distributed along the circumferential direction in one blade passage. Thus, the ratio of $W$ (the slot width) to $G$ (the gap between each slot pitch) is set to 2:1, i.e., the casing porosity $\Phi$ (the ratio of slot opening surface area to full annulus area within the slot axial extent) is $66.7 \%$. Fundamental design parameters of the baseline slot are listed in Table 2. Specifically, 
for BE slot, the first segment is designed to align with the axial direction, and the bending angle between the axial direction and the second segment $\beta$ roughly equals to the blade tip stagger angle (Zhu and $\mathrm{Chu}, 2005)$. For CU slot, the arc chord is also aligned with the axial direction. The rotor tip exposure $L_{e x}$ and casing porosity $\Phi$ are altered to study their influences on the casing treatment effectiveness, via upstream moving of the slot ending position and reducing the number of slots. The slots with $L_{e x}$ of $33.3 \%$ and $25 \%$ are denoted as CU1 and CU2. On the basis of CU2, the slots with reduced $\Phi$ are named as CU3 and CU4. The design parameters of four CU slots are presented in Table 3.

Table 2. Fundamental parameters of baseline slots

\begin{tabular}{|l|c|}
\hline \multicolumn{1}{|c|}{ Parameter [unit] } & Value \\
\hline \hline Slots/blades ratio $[-]$ & $5: 1$ \\
\hline Axial length of slot $L\left[\% c_{x, t i p}\right]$ & 79 \\
\hline Rotor tip axial chord exposure $L_{e x}\left[\% c_{x, t i p}\right]$ & 50 \\
\hline Slot width $W\left[\% c_{x, t i p}\right]$ & 24 \\
\hline Slot width/slot gap $W / G[-]$ & $2: 1$ \\
\hline Circumferential casing porosity $\Phi[\%]$ & 66.7 \\
\hline Slot radial depth $H\left[\% c_{x, t i p}\right]$ & 21.5 \\
\hline Radial skewed angle $\alpha\left[^{\circ}\right]$ & 60 \\
\hline
\end{tabular}

Table 3. Comparison of CU configurations

\begin{tabular}{|c|c|c|c|}
\hline Notation & $\begin{array}{c}L_{\text {ex }} \\
{\left[\% c_{x, \text { tip }}\right]}\end{array}$ & $\begin{array}{c}\text { Slots/blades } \\
\text { ratio [-] }\end{array}$ & $\begin{array}{c}\Phi \\
{[\%]}\end{array}$ \\
\hline \hline CU0 & 50 & $5: 1$ & 66.7 \\
\hline CU1 & 33.3 & $5: 1$ & 66.7 \\
\hline CU2 & 20 & $5: 1$ & 66.7 \\
\hline CU3 & 20 & $4: 1$ & 53.3 \\
\hline CU4 & 20 & $3: 1$ & 40 \\
\hline
\end{tabular}

\subsection{Numerical methods}

Commercial CFD solver ANSYS-CFX 14.5 has been employed for 3D steady-state calculations. Three-dimensional RANS equations have been discretized with the finite volume method. The two-equation $k$ - $\omega$ turbulence model has been used. For saving computation resources, single blade passage computation has been conducted. Block-structured grids are generated for blade passages, tip clearance region and casing treatment slots independently. The IGV, R1 and S1 grid consists of about 0.5 million cells per passage, with ATM topology, while the rotor tip clearance geometry is meshed in a simple H-type mesh with 11 cells in the radial direction to simulate tip leakage flow. The casing treatment grid consists of 18081 cells in the H-type mesh for each slot with 41 points in the axial direction and 21 points in both radial and circumferential directions. The grids are clustered at the solid wall to meet the resolution requirement of $y^{+} \leqslant 5$.

One blade passage is calculated with a periodic boundary condition in the circumferential direction. At the IGV inlet, the flow direction is assumed to be axial. A constant total pressure of $101325 \mathrm{~Pa}$ and total temperature of $288.15 \mathrm{~K}$ are applied. The static back pressure is imposed at conditions of radial equilibrium to vary the operating point and allow the flow field to develop unimpaired. A mixing-plane scheme is applied on IGV/R1 and R1/S1 interfaces. Since unsteady simulations are time consuming for various slots configurations, a steady frozen rotor model is applied on R1/CT interface. 
The grid independence tests and numerical method validations are carried out by NASA Stage 35 (Reid and Moore, 1978), in which the loading and critical flow phenomenon at the rotor tip is similar to the investigated transonic compressor stage. Figure 3 presents the comparison of stage performance at $100 \%$ design speed with the grids number of $0.3,0.5$ and 1.0 million cells per passage. All these three predicted results agree well with the experimental data. Note that report by Reid and Moore (1978) gives the stall point of Stage 35 as $18.26 \mathrm{~kg} / \mathrm{s}$. As stated by Chima (2009), the rotor is probably in stable rotating stall at this operating point. Therefore, a steady simulation hardly reaches this point.

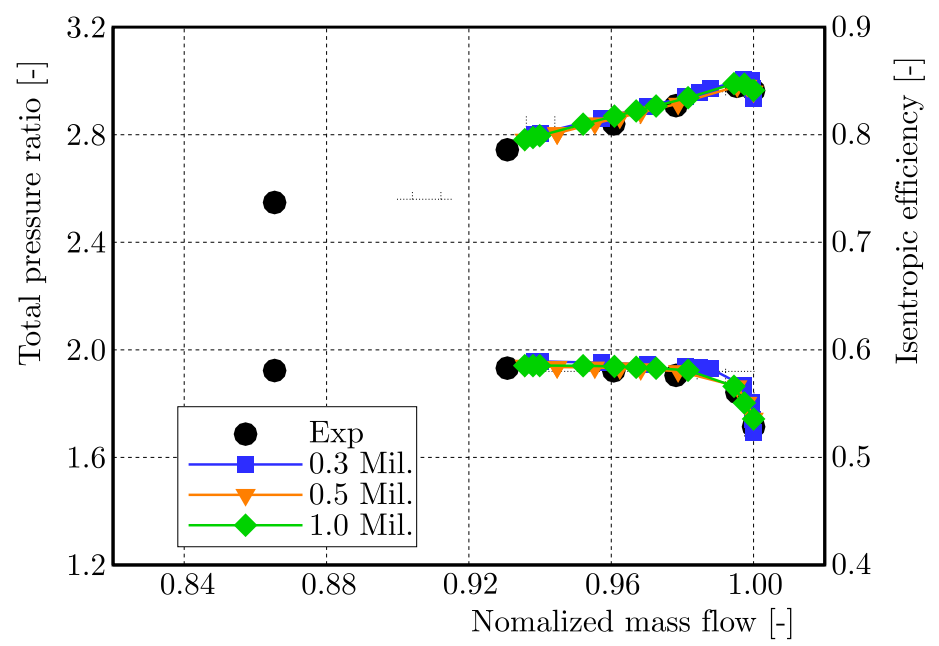

Fig. 3. Compressor maps of NASA Stage 35 (Reid and Moore, 1978) at 100\% design speed

Further comparisons of flow parameters are conducted at each peak efficiency point. The total pressure and temperature profiles downstream of rotor are presented in Fig. 4. Simulated profiles of different grid sets are all consistent with the experimental ones in trends. Curves of 0.5 and 1.0 million are rather close. But the total pressure ratio value is over-estimated across the whole span, especially below $40 \%$. While the simulated total temperature ratio profiles agree well with the experimental results. Convergence histories at the peak efficiency point are also presented in Fig. 5. RMS residuals of 0.3 and 0.5 million both descend below $10^{-6}$ after about 500 iterations, while 1.0 million grid set costs 800 iterations. Since 0.5 million is able to provide grid-independent and reliable solutions with much less computing resources, it is reasonable to use this grid set in this study.

\section{Results and discussions}

\subsection{Compressor characteristics}

Compressor speed lines with smooth casing (SC) and seven kinds of casing treatment (CT) are presented in Fig. 6. The total pressure ratio $\pi^{*}$ and isentropic efficiency $\eta^{*}$ are plotted as a function of mass flow $G . \pi^{*}$ and $\eta^{*}$ are normalized by each maximum value with SC, while $G$ is normalized by SC near the choked mass flow rate. The stall margin SM is calculated as follow

$$
\mathrm{SM}=\left(\frac{\pi_{\mathrm{NS}}^{*} / G_{\mathrm{NS}}}{\pi_{\mathrm{PE}}^{*} / G_{\mathrm{PE}}}-1\right) \cdot 100 \%
$$

where subscripts PE and NS represent the peak efficiency and near stall conditions.

According to Equation (3.1), this compressor stage has a SM of $6.67 \%$ at the specified rotating speed, which needs to be improved badly. The smooth casing peak efficiency (SCPE) and near 

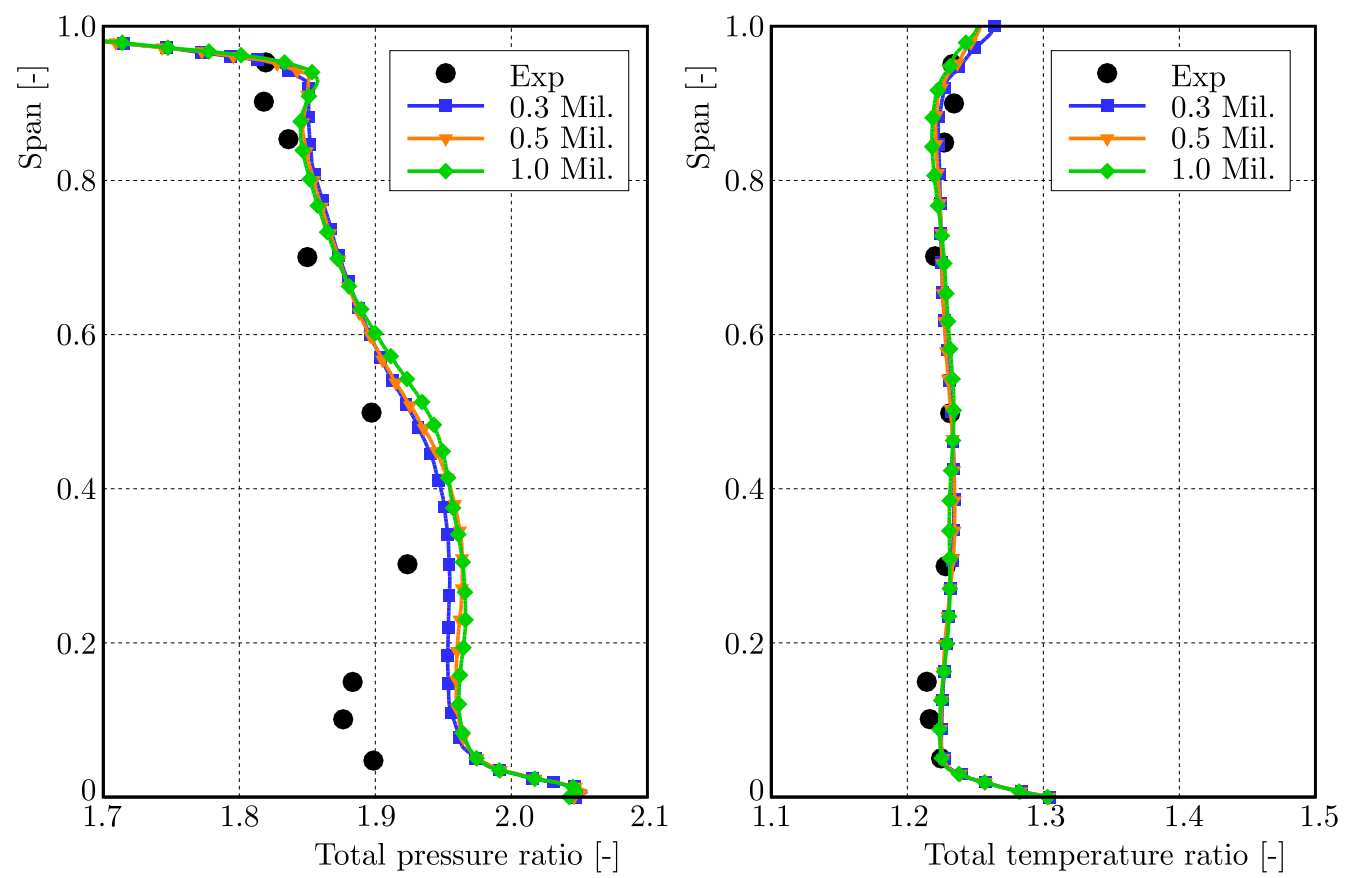

Fig. 4. Total pressure and temperature profiles downstream of Rotor 35 at the peak efficiency point

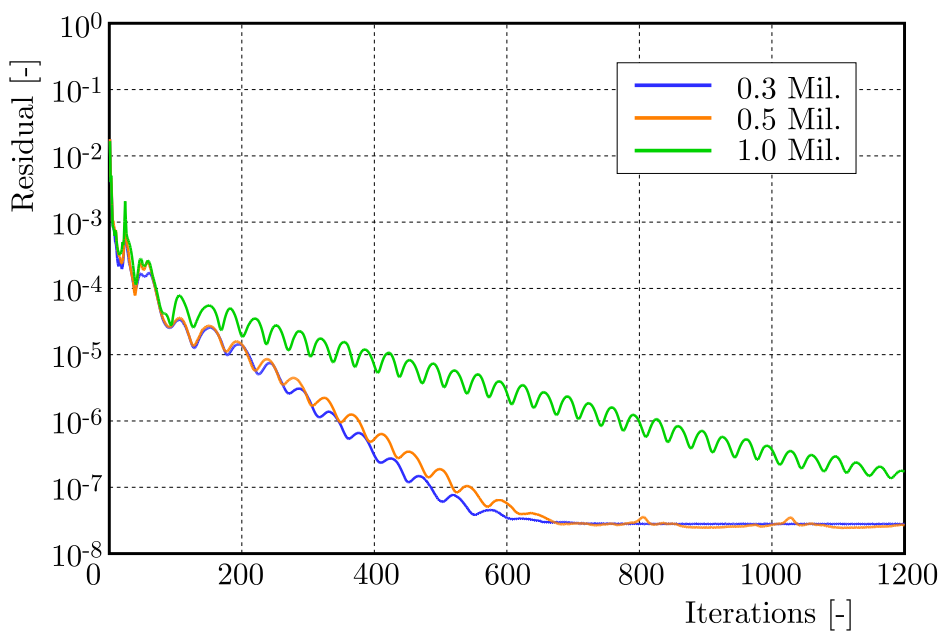

Fig. 5. Convergence histories at the peak efficiency point

stall (SCNS) points are located at $92.5 \%$ and $89.5 \%$ near the choked mass flow rate. Performance variations with various CTs at both SPCE and SCNS conditions are listed in Table 4. Steady results show that all slots with $50 \% L_{e x}$ are capable of extending SM by more than $20 \%$, with more than a $2 \%$ peak efficiency decrease. CU0 produces smaller $\Delta$ Eff compared with AX0 and BE0, along with $23 \% \Delta \mathrm{SM}$. As $L_{e x}$ is reduced from $50 \%$ (CU0) to $33.3 \%$ (CU1), both $\Delta \mathrm{SM}$ and $\Delta$ Eff drop significantly. Nevertheless, CU2 with $20 \% L_{e x}$ is able to provide similar $\Delta \mathrm{SM}$ and a less efficiency drop compared with CU1. As the slot number decreases from 5 (CU2) to 3 (CU4) per passage, the operating range declines along with smaller $\Delta$ Eff. Even no efficiency penalty is created by CU4 at SCNS condition.

\subsection{Entropy spanwise and streamwise distributions}

In this Section, pitch-averaged entropy distributions are analyzed to survey the development of loss generation with CTs. Entropy increase relative to incoming flow $(\Delta S)$ is calculated and normalized with the reference value $\Delta S_{\text {ref,sc }}$, the entropy value at $100 \%$ span with SC at SCPE condition. 

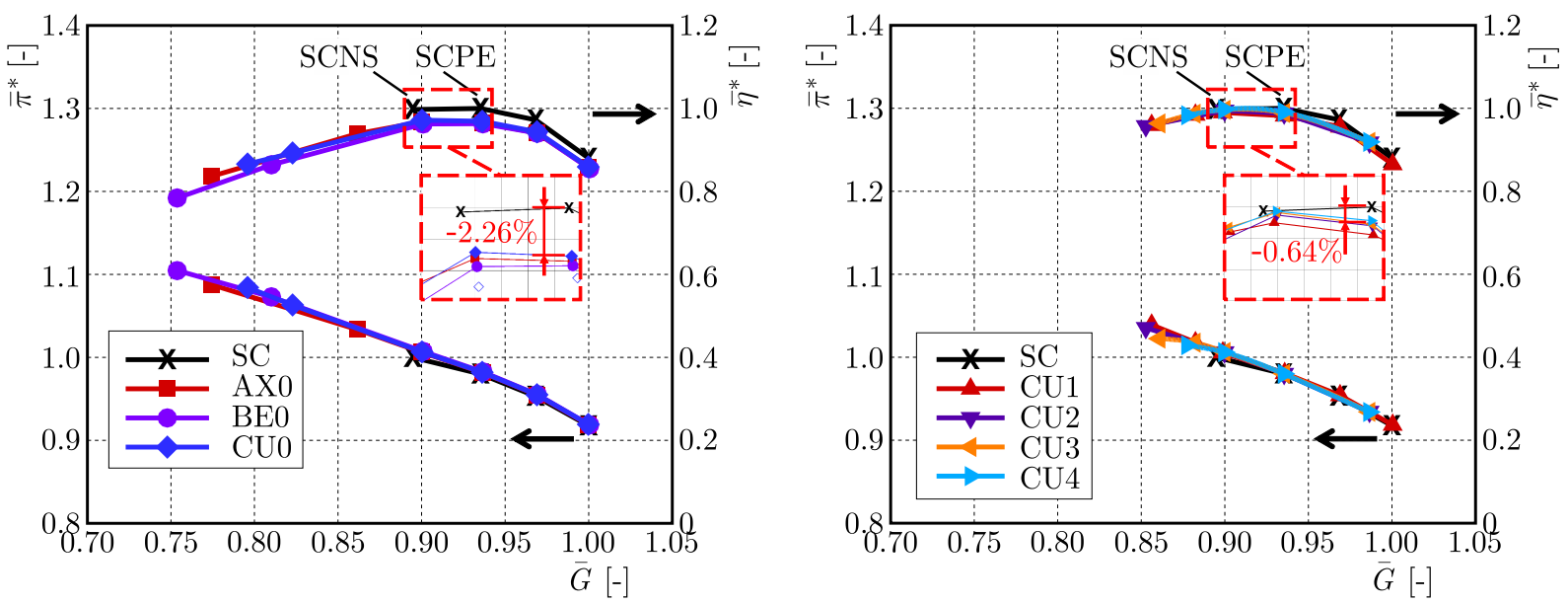

Fig. 6. Compressor maps with $\mathrm{SC}$ and three slot configurations

Table 4. Compressor performance variations with various CTs

\begin{tabular}{|c|c|c|c|}
\hline Notation & $\Delta$ SM [\%] & $\Delta$ Eff $_{\text {SCPE }}[\%]$ & $\Delta$ Eff $_{\text {SCNS }}[\%]$ \\
\hline \hline AX0 & 27.26 & 2.50 & 2.18 \\
\hline BE0 & 33.05 & 2.71 & 2.56 \\
\hline CU0 & 23.21 & 2.26 & 1.89 \\
\hline CU1 & 9.13 & 1.31 & 0.55 \\
\hline CU2 & 9.23 & 0.88 & 0.19 \\
\hline CU3 & 6.69 & 0.82 & 0.07 \\
\hline CU4 & 3.66 & 0.64 & 0.00 \\
\hline
\end{tabular}

Spanwise distributions of $\Delta S 20 \% c_{x, t i p}$ downstream the trailing edge (TE) at both conditions are presented in Fig. 7. At SCPE condition, as shown in Fig. 7 (a), $\Delta S$ with SC grows gradually from $90 \%$ span. While at SCNS condition, $\Delta S$ increases from $80 \%$ span at a higher growth rate. After the installation of slots, generally, $\Delta S$ span is altered only above $80 \%$ span. AX0 brought about loss growth from $80 \%$ to $95 \%$ span at both conditions, but has little impact near the rotor tip. BE0 and CU0 both shift $\Delta S$ to a higher level at both conditions, especially at the near tip regions. However, a remarkable entropy increase is merely found below $94 \%$ span with BE0/CU0 at SCNS condition, as shown in Fig. 7b. As for CU1 to CU4 slots, intensity and extent of effects on entropy distributions both decrease. It is worth noting that all four CUs are able to reduce entropy generation from $84 \%$ to $92 \%$ span at SCNS condition.

Since CTs have different influences on entropy spanwise distributions at different spans. The axial distributions of $\Delta S$ at two typical spans are presented to interpret the loss streamwise development. The $x$-coordinate represents the normalized axial position, while the $y$-coordinate represents $\Delta S / \Delta S_{r e f, s c}$. The axial range examined extends from $-30 \% c_{x, t i p}$ upstream LE to $20 \% c_{x, t i p}$ downstream TE.

Figure 8 presents axial distributions of $\Delta S$ at $90 \%$ span. As shown in Fig. 8 a, $\Delta S$ of SC rises gradually downstream LE at SCPE condition. While in Fig. 8b, $\Delta S$ curve shows a rapid growth downstream LE at SCNS condition. Slots of $L_{e x}=50 \%$ all raise the entropy value downstream $\mathrm{TE}$ at SCPE condition. The largest increase at the outlet is contributed by AX0, followed by BE0. However, a significant growth is not found within the coverage of slots, which indicates that the loss generation at this span does not directly result from the injection flow from slots. CUs with smaller extending length and slots number has little impact on $\Delta S$ distribution curves at SCPE condition. At SCNS condition, however, all slots bring down entropy curves within their extending ranges, except AX0. Only entropy value with AX0 is higher than SC case at the rotor outlet. CU0-CU4 even reduce the loss remarkably downstream TE. 
(a)

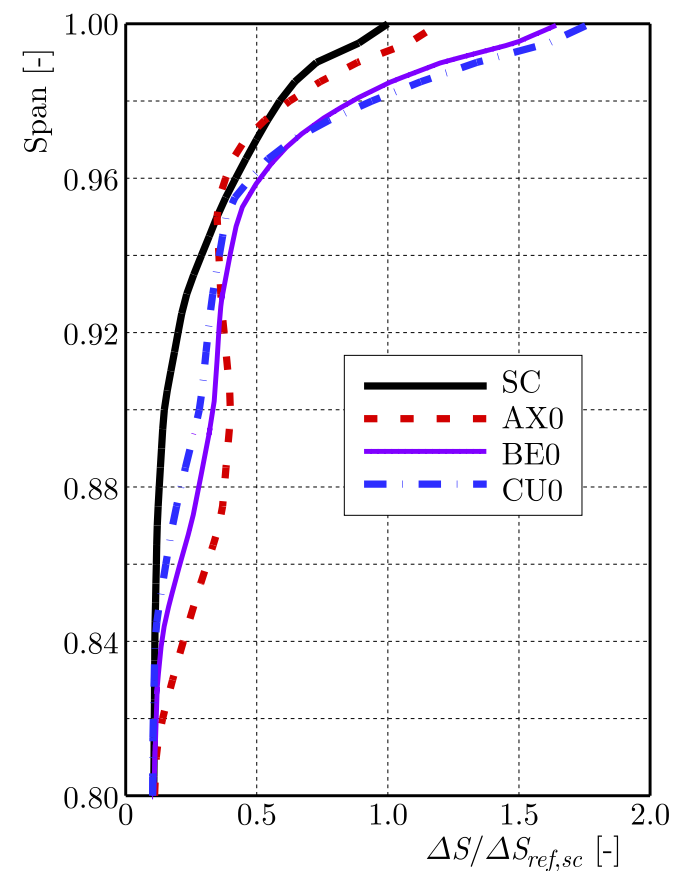

(b)

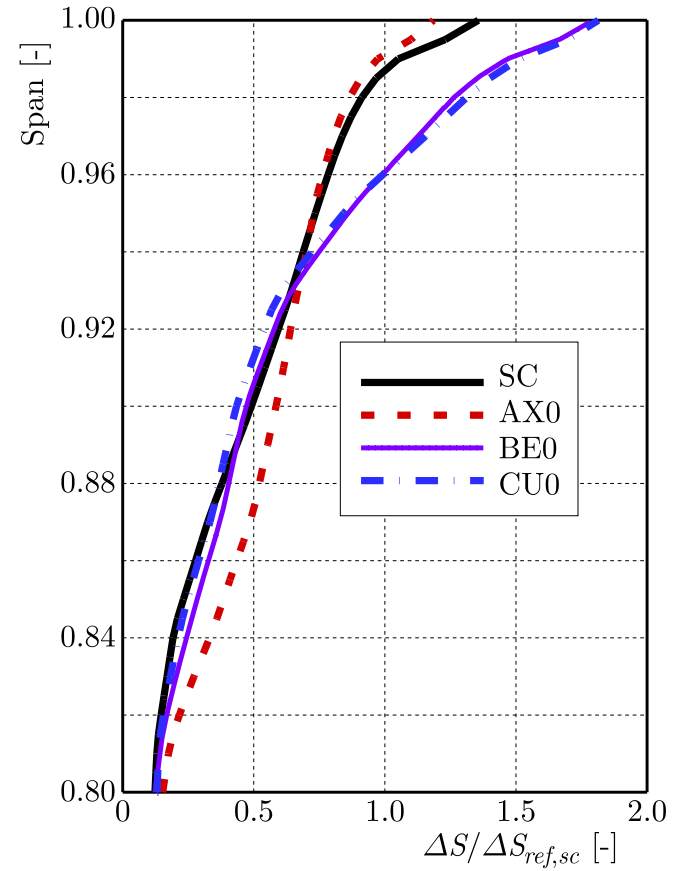

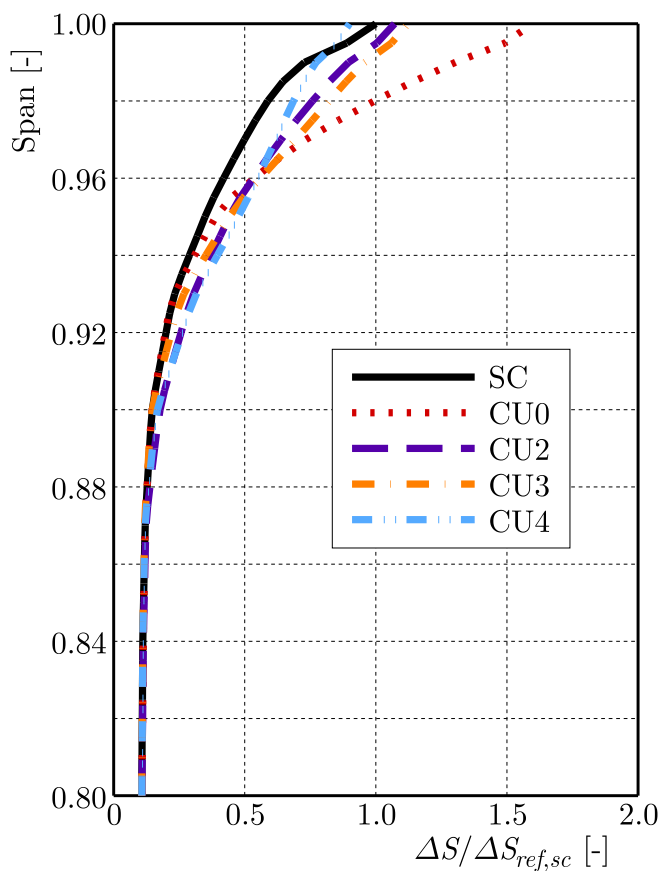

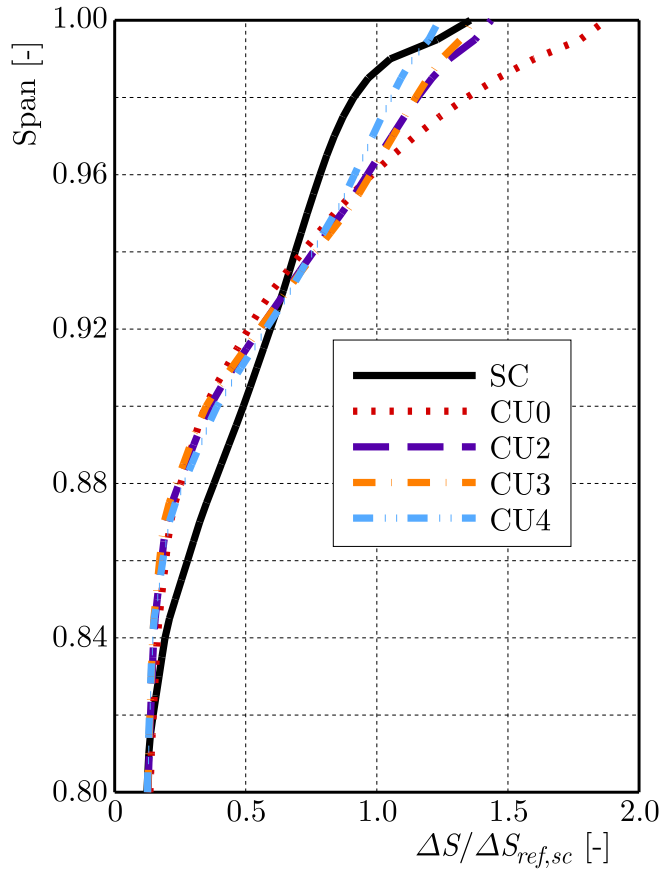

Fig. 7. Entropy distributions along the span at the rotor outlet at both conditions

Entropy streamwise distributions at 99.5\% span at SCPE condition are presented in Fig. 9a. $\Delta S$ of SC initiates rising gradually from $-10 \% x / c_{x, t i p}$ at SCPE condition. The maximum value is reached at $8 \% x / c_{x, t i p}$, followed by a tender decrease till $50 \% x / c_{x, t i p}$. After the installation of CTs, entropy begins to grow ahead of $-30 \% x / c_{x, t i p}$. For all 7 kinds of slots, local entropy increases at the fastest rate within the range from $-30 \%$ to $-20 \% x / c_{x, t i p}$. It is indicated that the injection procedure becomes the primary source of loss generation at $99.5 \%$ span. CU0 reaches the its peak $\Delta S$ value at about $15 \% x / c_{x, t i p}$. Being consistent with the spanwise distributions in Fig. 7a, entropy value of CU0 occupies the first place downstream TE; BE0 comes the second.

At SCNS condition, as shown in Fig. 9b, $\Delta S$ with SC starts to rise sharply from $-15 \%$ and reaches the peak value upstream LE. It results from forward motion of the interface between the incoming flow and the tip leakage flow. $\Delta S$ distributions with slots of $L_{e x}=50 \%$ vary slightly 
(a)

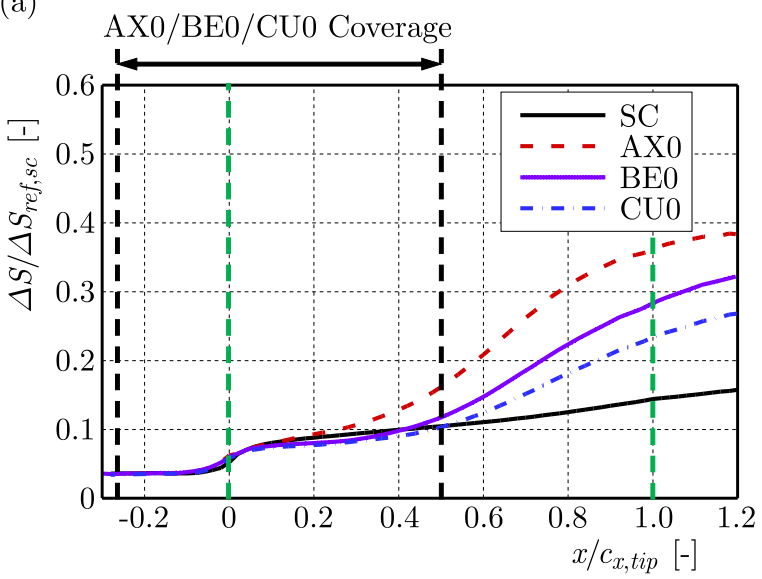

(b)

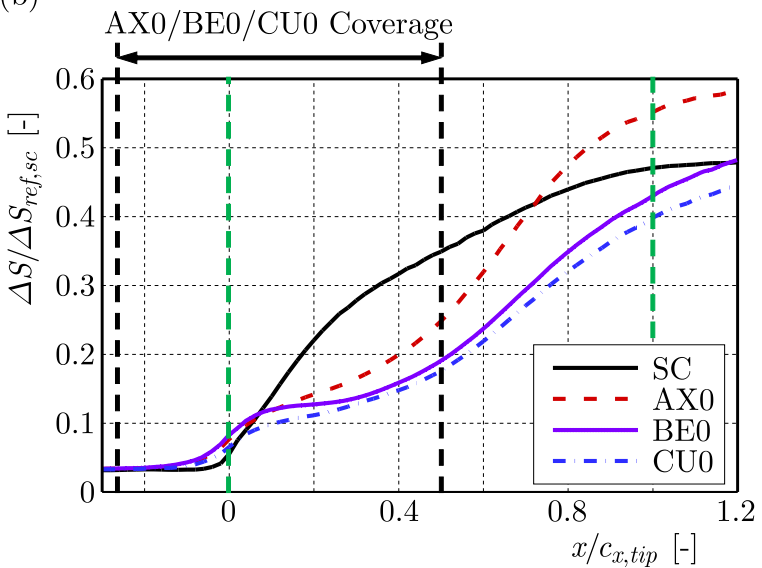

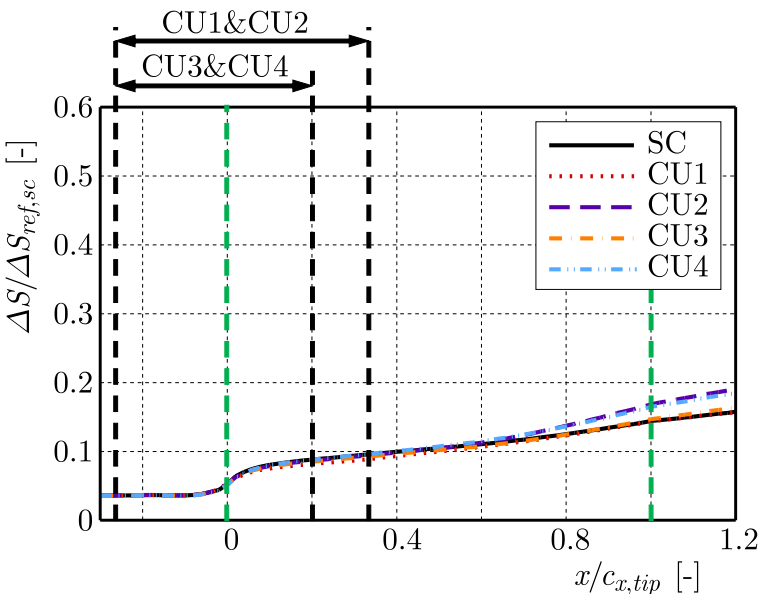

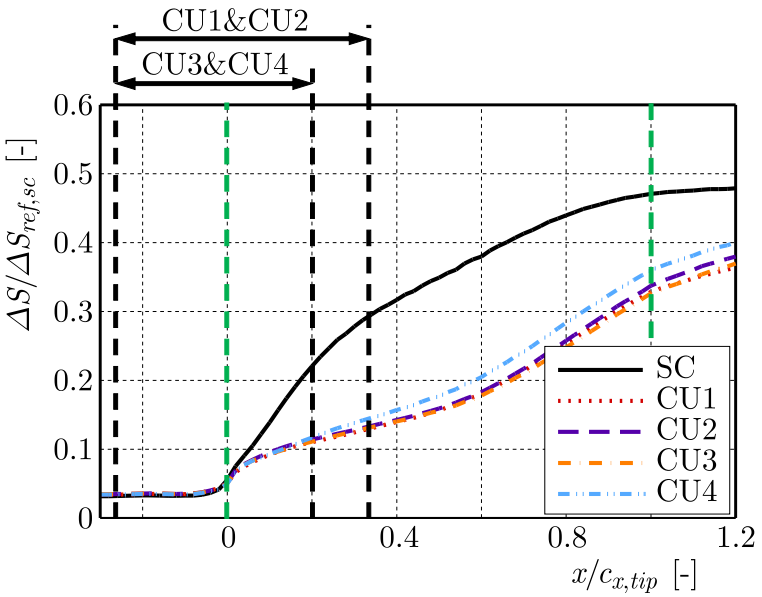

Fig. 8. Pitchwise-averaged entropy distributions along the axial direction at $90 \%$ span

compared to SCPE condition, in spite of a significant change in SC curve. But curves of CU1 to CU4 raise obviously compared to SCPE condition. It is indicated that slots with strong effects tend to maintain their influence on the flow filed at more critical conditions.

From the above analysis of entropy spanwise and streamwise distributions, it is concluded that the influenced spanwise range of entropy distributions by CTs is above $80 \%$ span, and the most dramatic growth generally occurrs above 95\% span. Primary sources of loss generation are different at lower and higher spans. At higher span high entropy regions are located within the slots axial coverage, which directly results from the injection flow by CTs. At a lower span, the entropy rises gradually along the streamwise direction. It is inferred that entropy variations are indirectly induced by an altered flow field by CTs. The following Section considers he loss development process in the main flow and inside slots, making further efforts to interpret the loss mechanisms with CU slots.

\subsection{Detailed Loss analysis with CU0}

This Section discusses the loss development process in the main flow and inside slots along the axial direction, making further efforts to interpret the loss mechanisms with CU0. Eight axial cuts are taken through the blade row and slots to show the increase in entropy relative to the inflow. The 8 cuts are plotted with CU0 slots in Fig. 10, with their normalized tip axial locations marked on the right side of the figure. The axial cuts and slots in one passage are numbered by Arabic and Roman numeral respectively, to facilitate the description in the following analysis. On these eight cuts, the normalized entropy distributions above about $80 \%$ span with SC at 
(a)

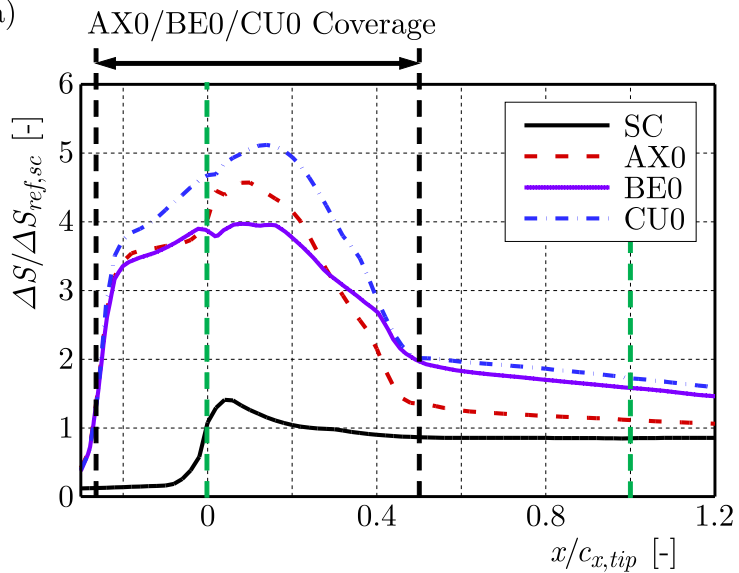

(b)

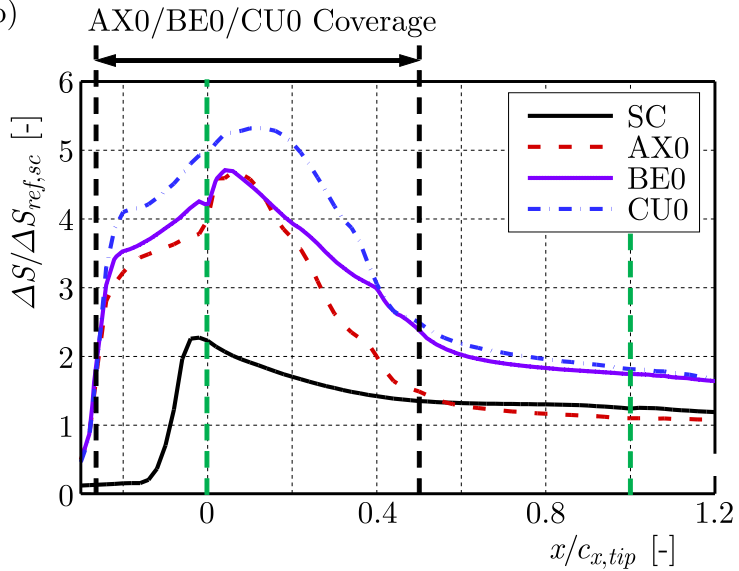

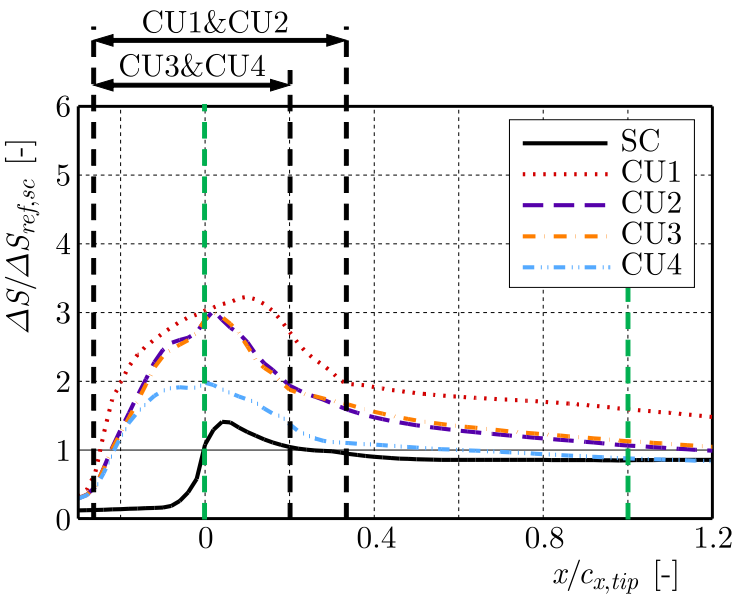

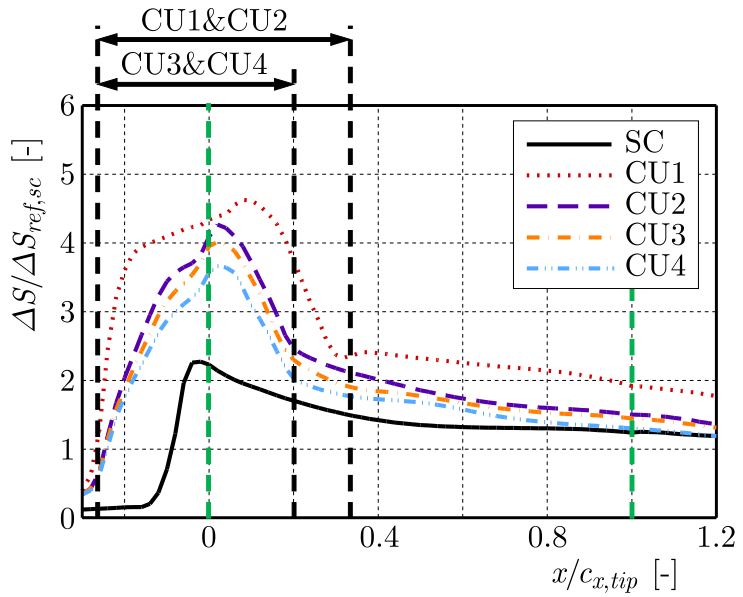

Fig. 9. Pitchwise-averaged entropy distributions along the axial direction at $99.5 \%$ span

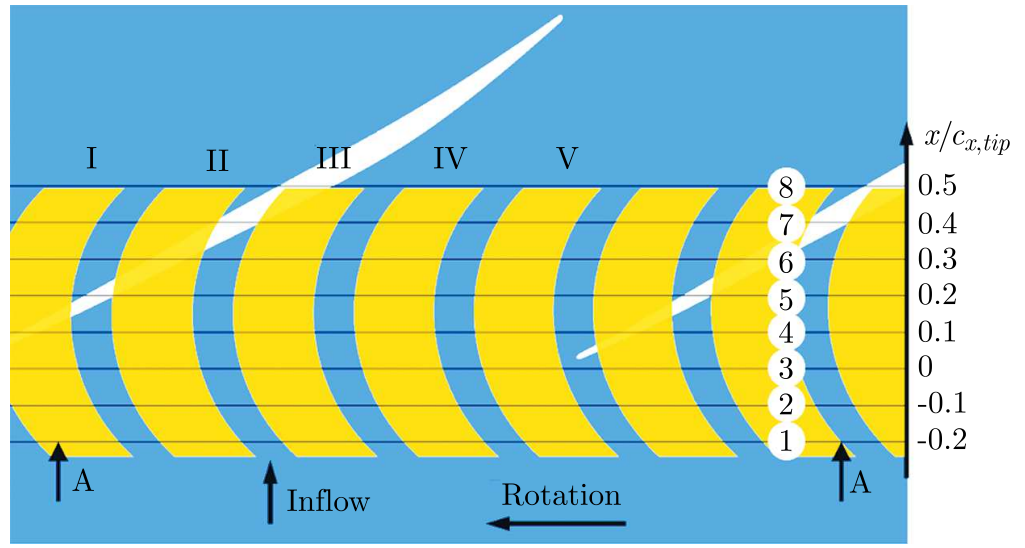

Fig. 10. The locations of eight axial cuts

both conditions are depicted in Fig. 12 (viewing from inlet to outlet). Figure 11 also presents the rotor blade limiting streamlines and 3D streamlines starting above $95 \%$ span in Cut 8 .

As shown in Fig. 12a, high entropy regions hardly appears in the first two cuts with SC. Then a small piece of a high loss region initiates to emerge near the casing in the 3rd cut, as a result of tip leakage flow spillage at LE. Besides, LE separation also gives rise to a relative high loss at lower span at the suction surface (SS). With the development of separation and tip leakage flow, high entropy regions near SS and casing extend in the main flow passage in following planes. At SCNS condition, the primary losses are still ascribed to the blade separation and tip leakage flow. However, since the tip leakage flow is spilling more upstream at this working condition, 
(a)

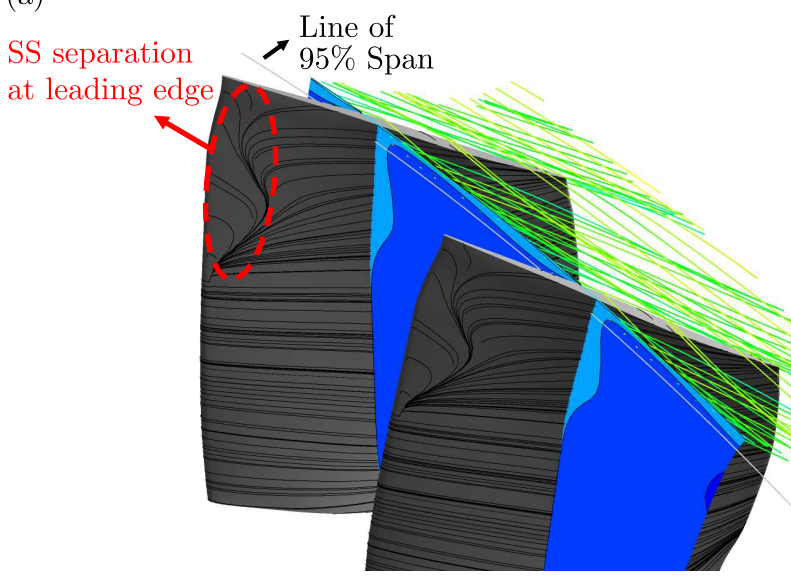

Velocity

- ^่

Fig. 11. Blade limiting streamlines and 3D streamlines starting above $95 \%$ span in Cut 8 (b)

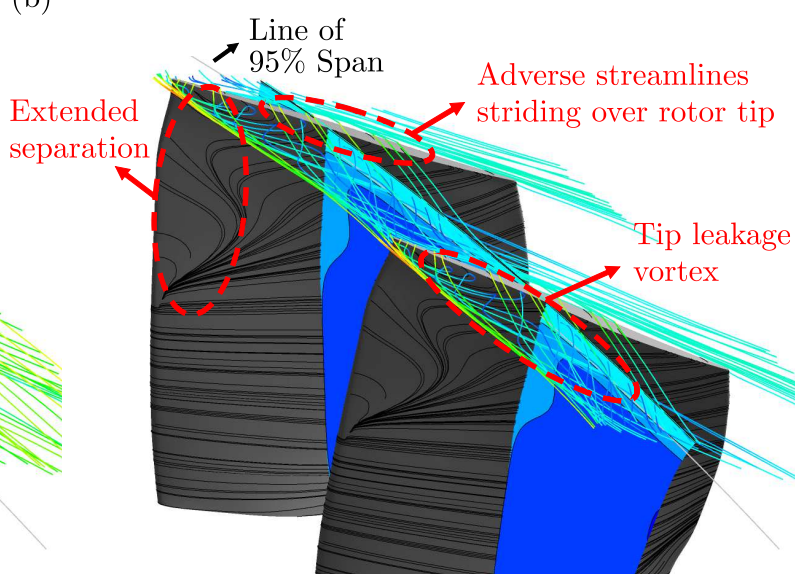

$\left[\mathrm{m} \mathrm{s}^{-1}\right]$

\section{$0^{\circ}$}
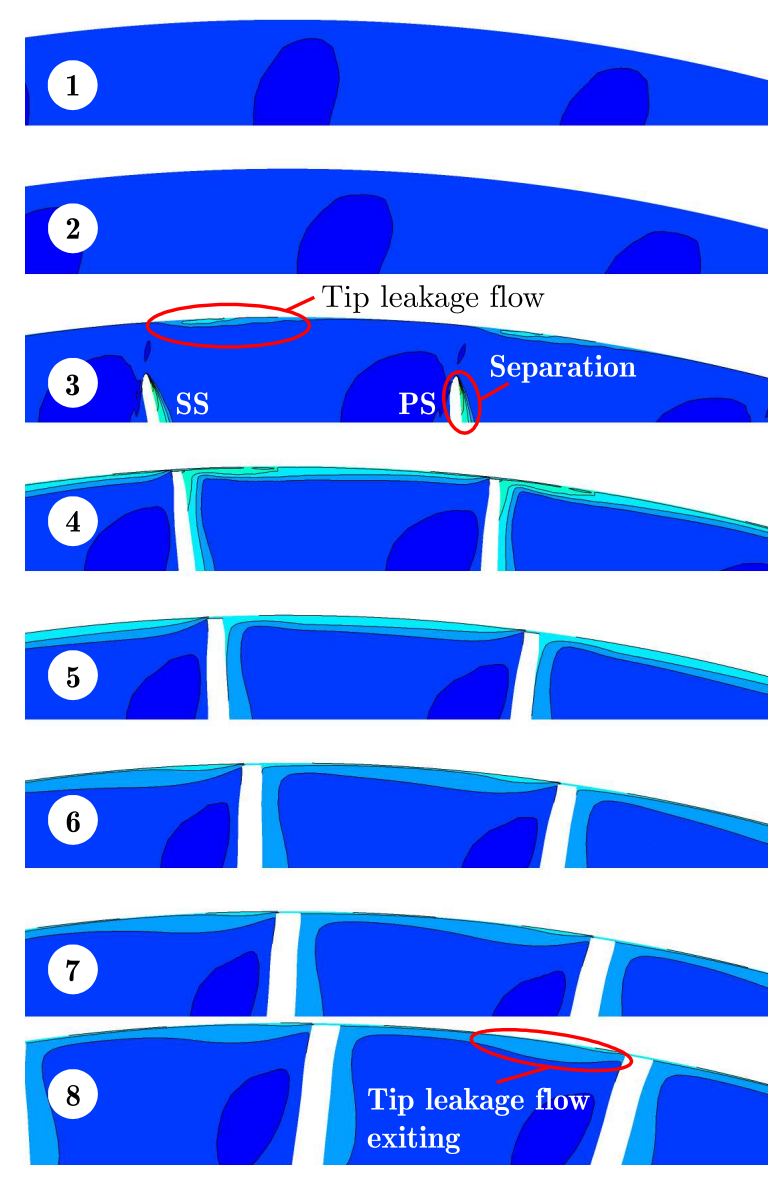

Fig. 12. Entropy distributions on axial cuts with SC (inlet to outlet)
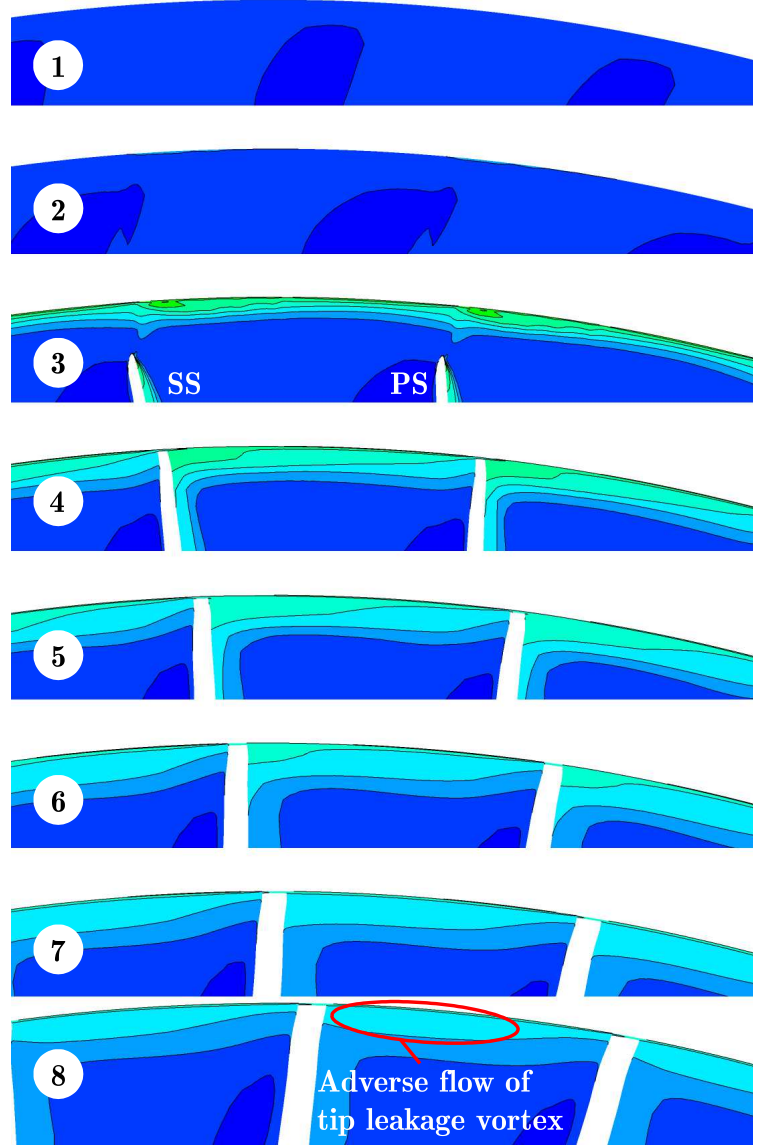
loss of much larger magnitude and extent is generated near the tip region, as shown at Cut 3 to Cut 9 in Fig. 12b. In addition, at SCNS condition, the high entropy region grows larger in the radial extent and moves closer to SS.

As seen in Fig. 11, the limiting streamlines at both two conditions clearly show the suction surface separation at LE. At SCNS condition, the separation region extends in both streamwise and spanwise directions. As shown in Fig. 11a, 3D streamlines just go downstream smoothly at a high speed at SCPE condition. Streamlines upstream Cut 8 are not observed. At SCNS condition, however, adverse streamlines striding over rotor tip are clearly seen upstream Cut 8 in Fig. 11b as well as a tip leakage vortex. This indicates that the high entropy region above $95 \%$ span in Cut 8 is attributed to the adverse flow. Streamlines starting from Cut 8 turn around and flow towards the pressure surface (PS) of the adjacent blade, as a result of a high adverse pressure gradient. Then streamlines strode over rotor tip and continue to propagate in pitchwise and reversed-stremwise directions. Some streamlines roll up and form a tip leakage vortex, consequently giving a rise to a larger area of the high entropy region at Cut 8 .

The axial distributions of pitch-averaged mass flow through the slot opening surface $\bar{G}_{\text {slot }}$ are depicted in Fig. 13. Values greater than zero represent the flow into the slot, and vice versa. Figure 14 presents the entropy distributions with CU0.
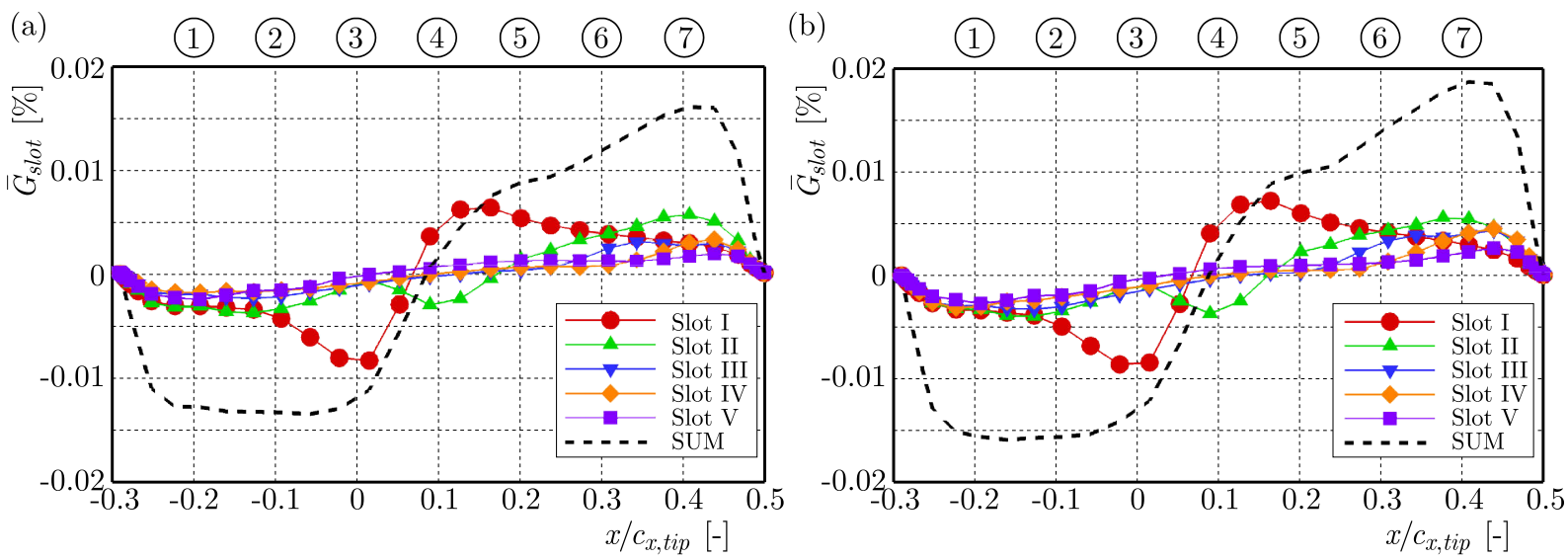

Fig. 13. Axial distributions of pitch-averaged mass flow through each slot opening surface

In Fig. 13, the sum of exchanging mass flow through five slots is also designated by dashed lines. Since the frozen rotor model has been used, the pattern of $\bar{G}_{\text {slot }}$ distribution is related to the relative position of R1/CU0. In this case, Slot I just strides over LE as shown in Fig. 10. Thus Slot I reaches its maximum injection mass flow rate at LE. The suction mass flow of Slot II reaches the maximum value at about $40 \% x / c_{x, \text { tip }}$, where Slot II overlaps with the blade tip. Since Slot III to V do not overlap with the blade tip, their distribution curves are similar. For the SUM curve, $10 \% x / c_{x, \text { tip }}$ is the dividing location of injection and suction regions. The injection flow rate is relatively large and almost constant from $-25 \%$ to $0 \% x / c_{x}$,tip. The suction flow rate increases downstream $10 \% x / c_{x, t i p}$ gradually and reaches the peak value at $40 \% x / c_{x, t i p}$. $\bar{G}_{\text {slot }}$ distributions at SCNS condition are similar with SCPE case.

In Fig. 14a, pretty high entropy regions are located on the counter-rotating side of Slot I (on the right side in current view point) at Cut 1-4. It is suggested that mixing of the injection flow and the main flow is the primary cause of the loss. The highest value of entropy appears at Cut 4, i.e. $10 \% x / c_{x, t i p}$ location, in accordance with the entropy axial distribution in Fig. 9a. Downstream $15 \% x / c_{x, t i p}$, the injection flow rate is no longer presented in any slot. Accordingly, the entropy value starts to decline as seen in Fig. 9a. At Cut 5-6, the upstream high entropy flow is absorbed into the rear portion of Slot I repeatedly. At Cut 8, SS separation area shrinks slightly. In addition, a noticeable high entropy region rises near casing and PS, which results from the accelerated tip flow by slots. The entropy distribution pattern is similar at SCNS 


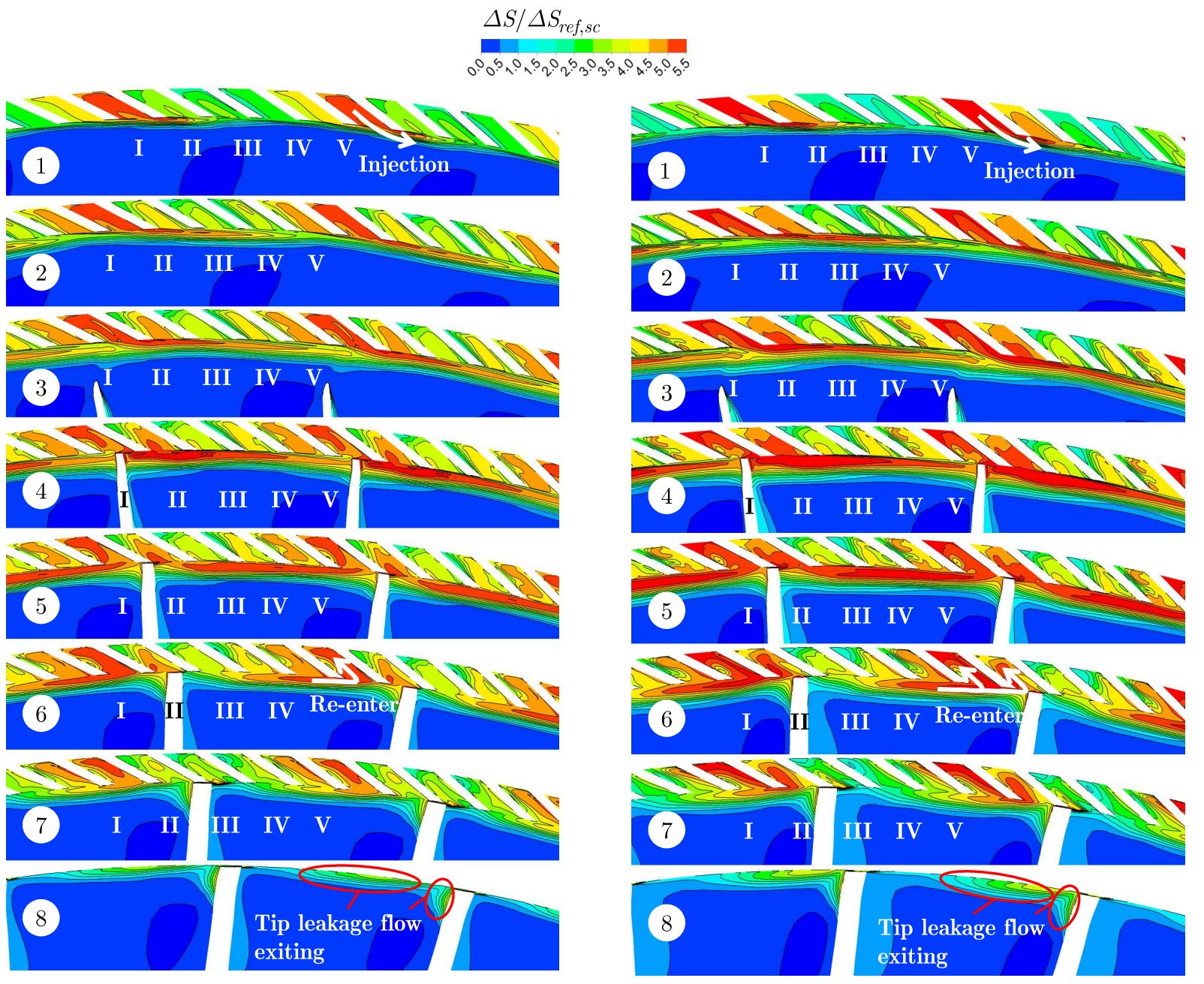

Fig. 14. Entropy distributions on axial cuts with CU0

condition as shown in Fig. 14b, with a slightly higher entropy level inside the slots and rotor passages.

From the above, loss development procedures throughout the passages with SC and CU0 are clarified. Loss generations with SC are primarily ascribed to the tip leakage flow and SS separation at LE. At a more critical condition, the rolled-up tip leakage vortex and consequent adverse flow occupy the major part of the high loss. CU0 is capable of suppressing SS separation loss, meanwhile accelerating the tip leakage flow and, consequently, bringing about an additional loss near the casing and PS. Besides, the upstream high entropy flow would be absorbed into the rear portion of the slot repeatedly, which results in further losses.

\section{Conclusions}

This paper clarifies the influence of the injection flow on loss distributions and elaborates the loss mechanisms. Several conclusions are drawn below:

- The influenced spanwise range of entropy distributions by slots is above $80 \%$ span. The most dramatic growth generally occurs above $95 \%$ span, which directly results from the injection flow upstream the leading edge. Entropy generation at a lower span rises gradually along the streamwise direction, which may be indirectly induced by an altered flow field by casing treatment. 
- Loss generations with smooth casing are primarily ascribed to the tip leakage flow and suction surface separation at the leading edge. At a more critical condition, the rolled-up tip leakage vortex and the consequent adverse flow occupy the major part of the high loss. CU0 slot is capable of suppressing the suction surface separation loss, meanwhile accelerating the tip leakage flow and, consequently, bringing about an additional loss near the casing and pressure surface. Besides, the upstream high entropy flow would be absorbed into the rear portion of the slot repeatedly, which results in further losses.

\section{Acknowledgements}

This work has been supported by the Natural Science Foundation of China (No. 51576124) and the "2011 Aero-Engine Collaborative Innovation Plan".

\section{References}

1. Alone D.B., Kumar S.S., Thimmaiah S.M., Mudipalli J.R.R., Pradeep A.M., RamaMURTHY S., IYENGaR V.S., 2014, Improvement of moderately loaded transonic axial compressor performance using low porosity bend skewed casing treatment, International Journal of Rotating Machinery, 2014, 1-14

2. Chima R.V., 2009, Swift code assessment for two similar transonic compressors, AIAA, 2009-1058

3. Danner F.C.T., Kau H.-P., Müller M.W., Schiffer H.-P., Brignole G.A., 2009, Experimental and numerical analysis of axial skewed slot casing treatments for a transonic compressor stage, ASME, GT2009-59647

4. FAn L., Ning F., LiU H., 2008, Aerodynamics of compressor casing treatment: part I - experiment and time-accurate numerical simulation, ASME, GT2008-51541

5. Fujita H., Takata H., 1984, A study on configurations of casing treatment for axial flow compressors, Bulletin of JSME, 27, 230, 1675-1681

6. Li M., Yuan W., Song X., Lu Y., Li Z., Li Q., 2012, An experimental investigation about the effect of the partial casing treatment on a transonic axial-flow compressor performance under inlet distortion, ASME, GT2012-68442

7. Lu X., Chu W., Zhu J., Wu Y., 2006, Experimental and numerical investigation of a subsonic compressor with bend skewed slot casing treatment, ASME, GT2006-90026

8. Moore R.D., Kovich G., Blade R.J., 1971, Effect of casing treatment on overall and blade element performance of a compressor rotor, NASA TN D-6538

9. Reid L., Moore D., 1978, Performance of a single-stage axial-flow transonic compressor with rotor and stator aspect ratios of 1.19 and 1.26, respectively, and with design pressure ratio 1.82 , NASA TP-1338

10. Takata H., Tsukuda Y., 1977, Stall margin improvement by casing treatment - its mechanism and effectiveness, Journal of Engineering for Power, 99, 1, 121-133

11. Tuo W., Lu Y., Yuan W., Zhou S., Li Q., 2011, Experimental investigation on the effects of unsteady excitation frequency of casing treatment on transonic compressor performance, Journal of Turbomachinery, 133, 2, 021014-6

12. YU Q., Li Q., Li L., 2002, The experimental researches on improving operating stability of a single stage transonic fan, ASME, GT2002-30640

13. ZHU J., ChU W., 2005, The effects of bend skewed groove casing treatment on performance and flow field near endwall of an axial compressor, AIAA, 2005-809 